\title{
Correlation-Function Identities and Inequalities for Ising Models with Pair Interactions
}

\author{
R. J. Boel and P. W. Kasteleyn \\ Instituut-Lorentz voor Theoretische Natuurkunde, Rijksuniversiteit Leiden, Leiden, The Netherlands
}

\begin{abstract}
For Ising models with pair interactions in zero magnetic field a class of linear combinations of products of two correlation functions is studied. We derive sufficient and necessary conditions under which a function in this class is (a) zero for all values of the coupling parameters, or (b) nonnegative for all nonnegative values of the coupling parameters. Examples of correlationfunction identities and inequalities of this type are given.
\end{abstract}

\section{Introduction}

In a recent paper [1], to be referred to as I, it was proved that for a (zero-field) Ising model on a planar graph the correlation functions for spins on a so-called boundary set satisfy certain algebraic relations which are valid for all values of the coupling parameters between the spins. These relations can all be derived from a set of identities which can be written in the form

$$
\sum_{j=1}^{n}(-1)^{j}\left\langle\sigma_{v_{1}} \sigma_{v_{j}}\right\rangle\left\langle\sigma_{v_{1}} \sigma_{v_{j}} \prod_{k=1}^{n} \sigma_{v_{k}}\right\rangle=0
$$

where $\left(v_{1}, \ldots, v_{n}\right)$ is a sequence of (not necessarily distinct) vertices which is a boundary sequence of the planar graph [cf. I Eq. (17)].

The left-hand side of the above equation can be considered as a special case of a function of the type $\sum_{B \subset A} \lambda_{B}\left\langle\sigma_{B}\right\rangle_{G}\left\langle\sigma_{B} \sigma_{D}\right\rangle_{G}$, where $G$ is an arbitrary graph, $A$ is an arbitrary set of vertices of $G, D$ a subset of $A$, the sum is over all subsets of $A$, and the coefficients $\lambda_{B}$ are independent of the coupling parameters; the case where all vertices in the boundary sequence are different then corresponds to the case $D=A$, i.e. $\sigma_{B} \sigma_{D}=\sigma_{A \backslash B}$.

In this paper we derive sufficient and necessary conditions on the coefficients $\lambda_{B}$ under which a function of this general type for an Ising model with pair interactions in zero magnetic field is (a) zero for all values of the coupling parameters, or (b) nonnegative for all nonnegative values of the coupling parameters, respectively. 
In Section 2 such conditions are derived for the case $D=A$ on the basis of an expansion of the Boltzmann factor with respect to the coupling parameters. They take, respectively, the form of a set of linear equations and a set of linear inequalities for the $\lambda_{B}$ with coefficients 0 and 1 . In Section 3 the results are extended to the general case $D \subset A$. Section 4 is devoted to an analysis of some properties of the sets of correlation-function identities that can be obtained in this way. In Section 5 various examples of such identities are given, among which those derived in I. In Section 6 we show that some known correlation-function inequalities follow from the general analysis given in this paper and we derive a new inequality. We end this paper with a few concluding remarks.

Since the extension of the partition function and the correlation functions of Ising models to complex values of the coupling parameters is sometimes useful, we allow for these complex values where possible. This implies that the partition function may take the value zero, in which case the normalized correlation functions are not defined. For this reason we shall work almost exclusively with unnormalized correlation functions; the translation of the results to normalized correlation functions is trivial.

\section{Conditions for the Existence of Certain Identities and Inequalities for Correlation Functions}

As in I we define a graph to be a pair $(V(G), E(G))$, where $V(G)$ is a set of elements called vertices and $E(G)$ a set of unordered pairs $\left\{v, v^{\prime}\right\}$ of distinct vertices, called edges. $G$ is finite if $V(G)$ and $E(G)$ are finite. For definitions used but not defined in this paper the reader is referred to [1] and [2].

An Ising model on a finite graph $G$ is defined as a triple $(G, \mathscr{S}, K)$, where $\mathscr{S}$ is the set of all functions $\sigma: V(G) \rightarrow\{-1,1\}$ (called configurations) and $K$ a complex function on $E(G)$ (called the interaction function). The spin variable $\sigma_{v}$ is the value of $\sigma$ at the vertex $v$, the coupling parameter $K_{e}$ is the value of $K$ at the edge $e$. The set of all interaction functions will be denoted by $\mathscr{K}$, the set of all $K \in \mathscr{K}$ such that $K_{e} \geqq 0\left(K_{e}>0\right)$ for all $e \in E(G)$ by $\mathscr{F}\left(\mathscr{F}^{+}\right)$; an Ising model $(G, \mathscr{S}, K)$ with $K \in \mathscr{F}\left(\mathscr{F}^{+}\right)$is called ferromagnetic (strictly ferromagnetic).

For any set $A \subset V(G)$ we define

$$
\sigma_{A}=\prod_{v \in A} \sigma_{v}
$$

for $A=\emptyset$ we have $\sigma_{\emptyset}=1$. For any edge $e=\left\{v, v^{\prime}\right\}$ and any set $X \subset E(G)$ we define

$$
\begin{aligned}
\sigma^{e} & =\sigma_{v} \sigma_{v^{\prime}} \\
\sigma^{X} & =\prod_{e \in X} \sigma^{e} .
\end{aligned}
$$

The Hamiltonian of an Ising model $(G, \mathscr{S}, K)$ is defined by

$$
H_{G, K}(\sigma)=-\sum_{e \in E(G)} K_{e} \sigma^{e}
$$

the unnormalized and normalized (spin) correlation functions $\left(\sigma_{A}\right)_{G, K}$ and $\left\langle\sigma_{A}\right\rangle_{G, K}$, respectively, for any set $A \subset V(G)$ by

$$
\left(\sigma_{A}\right)_{G, K}=\sum_{\sigma \in \mathscr{S}} \sigma_{A} \mathrm{e}^{-H_{G, K}(\sigma)},
$$




$$
\left\langle\sigma_{A}\right\rangle_{G, K}=\left(\sigma_{A}\right)_{G, K} Z^{-1} \quad \text { if } Z \neq 0,
$$

where $Z$, the canonical partition function, is defined by $Z=(1)_{G, K}$. For brevity, we shall often suppress the index $K$, and, where no confusion can arise, the index $G$ as well. We have taken $\beta=1$.

Since the Hamiltonian is quadratic in the $\sigma_{v}$, the correlation function $\left(\sigma_{A}\right)_{G, K}$ vanishes if $|A|$ is odd. Therefore, we shall henceforth consider only correlation functions $\left(\sigma_{A}\right)_{G, K}$ for even sets $A$, i.e. for sets with $|A|$ even.

We now consider an arbitrary even set $A \subset V(G)$. Let $\lambda=\left\{\lambda_{B}\right\}_{B \subset A}^{e}$ be a set of complex numbers defined for all even sets $B \subset A$, with the restriction $\lambda_{B}=\lambda_{A \backslash B}$ for all $B$. We introduce the following quadratic combination of unnormalized correlation functions:

$$
\Lambda_{A}(G, K)=\sum_{B \subset A}^{e} \lambda_{B}\left(\sigma_{B}\right)_{G, K}\left(\sigma_{A \backslash B}\right)_{G, K},
$$

where the sum is over all even sets $B C A$. For convenience, a function of the type (6) will be referred to as a $\Lambda$-function. We shall now derive a condition for $\lambda$ under which a $\Lambda$-function satisfies the equation $\Lambda_{A}(G, K)=0$ for all $K \in \mathscr{K}$ (to be referred to as a $\Lambda$-identity), and a condition for $\lambda$ under which it satisfies the inequality $\Lambda_{A}(G, K)$ $\geqq 0$ for all $K \in \mathscr{F}$ (to be referred to as a $\Lambda$-inequality).

We first consider an arbitrary product of two unnormalized correlation functions $\left(\sigma_{B}\right)_{G}\left(\sigma_{C}\right)_{G}$ with $B, C \subset V(G), B \cap C=\emptyset$. If we expand the Boltzmann factors in this product with the aid of the elementary relation

$$
e^{K_{e} \sigma^{e}}=c_{e}+s_{e} \sigma^{e},
$$

where $e$ is an arbitrary edge, $c_{e}=\cosh K_{e}$ and $s_{e}=\sinh K_{e}$, we obtain

$$
\left(\sigma_{B}\right)_{G}\left(\sigma_{C}\right)_{G}=\left\{c_{e}\left(\sigma_{B}\right)_{G^{\prime}}+s_{e}\left(\sigma_{B} \sigma^{e}\right)_{G^{\prime}}\right\}\left\{c_{e}\left(\sigma_{C}\right)_{G^{\prime}}+s_{e}\left(\sigma_{C} \sigma^{e}\right)_{G^{\prime}}\right\},
$$

where $\left(\sigma_{B}\right)_{G^{\prime}}$ etc. are correlation functions of the Ising model $\left(G^{\prime}, \mathscr{S}, K^{\prime}\right)$ obtained from $(G, \mathscr{S}, K)$ by deleting $e$ from $G$ and restricting $K$ to $E(G) \backslash e$ [which is equivalent to putting $K_{e}=0$ in $\left.H_{G, K}(\sigma)\right]$.

Using $c_{e}^{2}=1+s_{e}^{2}$ we rewrite Equation (8) as

$$
\begin{aligned}
\left(\sigma_{B}\right)_{G}\left(\sigma_{C}\right)_{G}= & \left(\sigma_{B}\right)_{G^{\prime}}\left(\sigma_{C}\right)_{G^{\prime}}+c_{e} s_{e}\left\{\left(\sigma_{B} \sigma^{e}\right)_{G^{\prime}}\left(\sigma_{C}\right)_{G^{\prime}}+\left(\sigma_{B}\right)_{G^{\prime}}\left(\sigma_{C} \sigma^{e}\right)_{G^{\prime}}\right\} \\
& +s_{e}^{2}\left\{\left(\sigma_{B}\right)_{G^{\prime}}\left(\sigma_{C}\right)_{G^{\prime}}+\left(\sigma_{B} \sigma^{e}\right)_{G^{\prime}}\left(\sigma_{C} \sigma^{e}\right)_{G^{\prime}}\right\} .
\end{aligned}
$$

We now repeat this process for all other edges of $G$. To write the result in a compact form we associate with each term in the resulting expression a function

$$
\theta: E(G) \rightarrow\{0,1,2\},
$$

where $\theta_{e}=0,1,2$ labels the first, second and third term in the right-hand side of Equation ( $\left.8^{\prime}\right)$, respectively, and the edge sets $L_{\theta}=\left\{e \in E(G) \mid \theta_{e}=1\right\}$ and $M_{\theta}=\left\{e \in E(G) \mid \theta_{e}=2\right\}$. The set of all functions $\theta$ is denoted by $\Theta$. We further define

$$
g(\theta)=\prod_{e \in E(G)} g_{e}\left(\theta_{e}\right),
$$

where $g_{e}(0)=1, g_{e}(1)=c_{e} s_{e}, g_{e}(2)=s_{e}^{2}$, and

$$
\Gamma_{\theta}(B, C)=\sum_{X \subset L_{\theta}} \sum_{Y \subset M_{\theta}}\left(\sigma_{B} \sigma^{X} \sigma^{Y}\right)_{G_{\theta}}\left(\sigma_{C} \sigma^{L_{\theta} \backslash X} \sigma^{Y}\right)_{G_{\theta}},
$$


in which $G_{\emptyset}$ is the graph defined by $V\left(G_{\emptyset}\right)=V(G), E\left(G_{\emptyset}\right)=\emptyset$.

The result of applying Equation (7) to all edges of $G$ is

$$
\left(\sigma_{B}\right)_{G}\left(\sigma_{C}\right)_{G}=\sum_{\theta \in \Theta} g(\theta) \Gamma_{\theta}(B, C) .
$$

A convenient way to characterize the various terms in $\Gamma_{\theta}(B, C)$ is the following. We define for each $\theta$ a graph $G_{\theta}$ by $V\left(G_{\theta}\right)=V(G), E\left(G_{\theta}\right)=L_{\theta} \cup M_{\theta}$; i.e. $G_{\theta}$ is obtained from $G$ by deleting all edges $e$ with $\theta_{e}=0$. For each pair of sets $X \subset L_{\theta}$, $Y \subset M_{0}$ we define a function

$$
\phi: E\left(G_{\theta}\right) \rightarrow \mathbb{Z}_{2} \times \mathbb{Z}_{2},
$$

where $\mathbb{Z}_{2}$ is the field of integers modulo 2 , by

$$
\phi_{e}=\left\{\begin{array}{lll}
(1,0) & \text { if } & e \in X \\
(0,1) & \text { if } & e \in L_{\theta} \backslash X \\
(1,1) & \text { if } & e \in Y \\
(0,0) & \text { if } & e \in M_{\theta} \backslash Y .
\end{array}\right.
$$

Conversely, every function of the type (13) with the property

$$
\phi_{e}=\left(\alpha_{e}, \beta_{e}\right) \text { with }\left\{\begin{array}{lll}
\alpha_{e}+\beta_{e}=1 & \text { if } & e \in L_{\theta} \\
\alpha_{e}+\beta_{e}=0 & \text { if } & e \in M_{\theta}
\end{array}\right.
$$

defines two sets $X \subset L_{\theta}, Y \subset M_{\theta}$, and hence a term in $\Gamma_{\theta}(B, C)$, by

$$
\begin{gathered}
X=\left\{e \in L_{\theta} \mid \phi_{e}=(1,0)\right\}, \\
Y=\left\{e \in M_{\theta} \mid \phi_{e}=(1,1)\right\} .
\end{gathered}
$$

We remark that in the terminology of algebraic graph theory $\phi$ is a 1-chain of $G_{\theta}$ over $\mathbb{Z}_{2} \times \mathbb{Z}_{2}$ (cf. [3]).

Let us now analyse the various terms of $\Gamma_{\theta}(B, C)$. Since $\left(\sigma_{A}\right)_{G_{\theta}}=\sum_{\sigma} \sigma_{A}$ and $\sum_{\sigma_{v}= \pm 1} \sigma_{v}=0$, we have

$$
\left(\sigma_{A}\right)_{G_{\theta}}=2^{|V(G)|} \delta_{A, \theta} \quad \text { for all } A \subset V(G) .
$$

Hence, the only nonvanishing terms in the right-hand side of Equation (11) are those for which

$$
\sigma_{B} \sigma^{X} \sigma^{Y}=\sigma_{\mathfrak{\vartheta}}, \quad \sigma_{C} \sigma^{L_{\theta} \backslash X} \sigma^{Y}=\sigma_{\mathfrak{\natural}} .
$$

The first condition requires that every vertex in $B$ is incident with an odd number of edges of $X \cup Y$ and every vertex in $V(G) \backslash B$ with an even number of edges of $X \cup Y$; the second condition is analogous.

These conditions can easily be translated into a condition on the function $\phi$ if we introduce the functions $\partial \phi: V(G) \rightarrow \mathbb{Z}_{2} \times \mathbb{Z}_{2}$ defined by

$$
(\partial \phi)_{v}=\sum_{e \text { inc } v} \phi_{e},
$$


where the sum (taken in $\mathbb{Z}_{2} \times \mathbb{Z}_{2}$ ) is over all edges in $G_{\theta}$ incident with the vertex $v$, and $\chi(B, C): V(G) \rightarrow \mathbb{Z}_{2} \times \mathbb{Z}_{2}$ defined by

$$
\chi_{v}(B, C)=\left\{\begin{array}{lll}
(1,0) & \text { if } & v \in B \\
(0,1) & \text { if } & v \in C \\
(0,0) & \text { if } & v \notin B \cup C .
\end{array}\right.
$$

$\partial \phi$ and $\chi(B, C)$ are 0 -chains of $G_{\theta}$ over $\mathbb{Z}_{2} \times \mathbb{Z}_{2}, \partial \phi$ is called the boundary of $\phi$. In terms of these functions, Equation (18) can be written as

$$
\partial \phi=\chi(B, C) .
$$

The set of all functions $\phi$ which, for a given choice of $\theta, B$ and $C(B \cap C=\emptyset)$, satisfy Equations (15) and (21) will be denoted by $\Phi_{\theta}(B, C)$. Since every non-vanishing term in the right-hand side of Equation (11) is equal to $2^{2|V(G)|}$ we have

$$
\Gamma_{\theta}(B, C)=2^{2|V(G)|}\left|\Phi_{\theta}(B, C)\right| .
$$

We now proceed to derive a few properties of the sets $\Phi_{\theta}(B, C)$.

We call a set $S$ of edges of a graph $G$ a (generalized) cycle of $G$ if each vertex of $G$ is incident with an even number of edges of $S$. The total number of cycles of $G_{\theta}$, including the empty cycle $\emptyset$, will be denoted by $\gamma_{\theta}$.

Lemma 1. If, for $\theta \in \Theta$ and for disjoint sets $B, C \subset V(G), \Phi_{\theta}(B, C)$ is not empty, then $\left|\Phi_{\theta}(B, C)\right|=\gamma_{\theta}$.

Proof. Let $\phi \in \Phi_{\theta}(B, C), S \subset E\left(G_{\theta}\right)$, and define $\phi^{\prime}=\phi+\psi$, where

$$
\psi_{e}=\left\{\begin{array}{lll}
(1,1) & \text { if } & e \in S \\
(0,0) & \text { if } & e \notin S
\end{array}\right.
$$

Then it is easily verified that $\phi^{\prime} \in \Phi_{\theta}(B, C)$ if and only if $S$ is a cycle of $G_{\theta}{ }^{1}$. Since this establishes a one-to-one correspondence between the functions $\phi^{\prime}$ in $\Phi_{\theta}(B, C)$ and the cycles $S$ of $G_{\theta}$, the lemma follows.

For any graph $G$ and any set $A \subset V(G)$ let $\pi(A, G)$ denote the partition of $A$ induced by $G$, i.e. the partition in which two vertices of $A$ are in the same block if and only if they are in the same connected component of $G$. If $H$ is a spanning subgraph of $G$, i.e. if $V(H)=V(G), E(H) \subset E(G)$, the partition $\pi(A, H)$ is a refinement of $\pi(A, G)$, i.e., the blocks of $\pi(A, H)$ are subsets of those of $\pi(A, G)$. If $H$ is a subgraph of $G$ such that $\pi(A, H)=\pi(A, G)$ and no proper subgraph of $H$ has this property, we call $H$ a skeleton graph associated with the partition $\pi(A, G)$. Evidently, a skeleton graph is a forest, i.e., it contains no circuits. It is easily seen that for each partition $\pi(A, G)$ with $A \neq \emptyset$ there is at least one skeleton graph.

The set of all partitions of $A$ will be denoted by $\Pi_{A}$, the set of all even partitions of $A$ (i.e. partitions of $A$ into even subsets) by $\Pi_{A}^{e}$, and the set of all even partitions of $A$ induced by spanning subgraphs of $G$ by $\Pi_{A}^{e}(G)$. Obviously, $\Pi_{\emptyset}=\Pi_{\emptyset}^{e}=\Pi_{\emptyset}^{e}(G)$ $=\emptyset$.

1 If $S$ is a cycle of $G_{\theta}, \psi$ is a cycle vector of $G_{\theta}$ [3]. If we denote the number of independent cycle vectors (the cycle rank or cyclomatic number) of a graph $G$ by $c(G)$, we have $\gamma_{\theta}=2^{c\left(G_{\theta}\right)}$. It is well known that $c(G)=|E(G)|-|V(G)|+$ number of connected components of $G$ 
Lemma 2. If, for $\theta \in \Theta$ and for disjoint sets $B, C \subset V(G), \Phi_{\theta}(B, C)$ is not empty, then $\pi\left(B, G_{\theta}\right)$ and $\pi\left(C, G_{\theta}\right)$ are even partitions.

Proof. Let $H$ be any connected component of $G_{\theta}, B_{H}=B \cap V(H)$, and $C_{H}$ $=C \cap V(H)$. Consider a function $\phi \in \Phi_{\theta}(B, C)$. By Equation (21) we have

$$
\sum_{v \in V(H)}(\partial \phi)_{v}=\sum_{v \in V(H)} \chi_{v}(B, C)=\sum_{v \in B_{H}}(1,0)+\sum_{v \in C_{H}}(0,1) .
$$

On the other hand, we have, by the definition of $\partial \phi$,

$$
\sum_{v \in V(H)}(\partial \phi)_{v}=\sum_{v \in V(H)} \sum_{e \text { inc } v} \phi_{e}=(0,0)
$$

since every edge in $H$ is counted twice in the double sum. It follows that $\left|B_{H}\right|$ and $\left|C_{H}\right|$ are even. Since $H$ is arbitrary, the lemma follows.

Lemma 3. If, for $\theta \in \Theta$ and for disjoint sets $B, C \subset V(G), \Phi_{\theta}(\emptyset, B \cup C)$ is not empty and $\pi\left(B, G_{\theta}\right)$ is an even partition, then $\Phi_{\theta}(B, C)$ is not empty.

Proof. Suppose that $\Phi_{\theta}(\emptyset, B \cup C)$ is not empty and that $\pi\left(B, G_{\theta}\right)$ is an even partition.

Consider a skeleton graph $H$ associated with $\pi\left(B, G_{\theta}\right)$. The set $E(H)$ is the disjoint union of two uniquely determined sets $E_{1}$ and $E_{2}$ with the property that if an edge $e \in E_{1}\left(e \in E_{2}\right)$ is deleted from $H$ the connected component of $H$ containing $e$ breaks up into two components, each one containing an odd (even) number of vertices of $B$. Each vertex in $B$ is incident with an odd number of edges of $E_{1}$, each vertex in $V(H) \backslash B$ is incident with an even number of edges of $E_{1}$. We now delete the edges of $E_{2}$ from $H$; in the resulting graph $H^{\prime}$ the vertices of $B$ are the only vertices of odd valency.

For $v \in B$ we have $\chi_{v}(\emptyset, B \cup C)=(0,1), \chi_{v}(B, C)=(1,0)$ and hence

$$
\chi_{v}(B, C)=\chi_{v}(\emptyset, B \cup C)+(1,1) .
$$

Consider a function $\phi \in \Phi_{\theta}(\emptyset, B \cup C)$. We define a function $\phi^{\prime}: E\left(G_{\theta}\right) \rightarrow \mathbb{Z}_{2} \times \mathbb{Z}_{2}$ by

$$
\begin{aligned}
& \phi_{e}^{\prime}=\phi_{e}+(1,1) \quad \text { if } \quad e \in E_{1}, \\
& \phi_{e}^{\prime}=\phi_{e} \text { if } \quad e \in E\left(G_{\theta}\right) \backslash E_{1} .
\end{aligned}
$$

Obviously $\phi^{\prime}$ satisfies Equation (15); by the above-mentioned property of $H^{\prime}$ it also satisfies Equation (21). Hence $\phi^{\prime} \in \Phi_{\theta}(B, C)$, which proves the lemma.

Let, for $A \subset V(G)$ and $\pi \in \Pi_{A}^{e}(G), \Theta_{\pi}(A)$ denote the set of all functions $\theta \in \Theta$ such that $\pi\left(A, G_{\theta}\right)=\pi$ and $\Phi_{\theta}(\emptyset, A)$ is not empty.

Lemma 4. For any $A \subset V(G)$, and any $\pi \in \Pi_{A}^{e}(G), \Theta_{\pi}(A)$ is not empty.

Proof. If $\Pi_{A}^{e}(G)$ is empty, the lemma is trivial, so let $\Pi_{A}^{e}(G)$ be non-empty, and $\pi \in \Pi_{A}^{e}(G)$. Then, by the definition of $\Pi_{A}^{e}(G)$, there is a spanning subgraph $G^{\prime}$ of $G$ such that $\pi\left(A, G^{\prime}\right)=\pi$. Let $H$ be a skeleton graph associated with $\pi\left(A, G^{\prime}\right)$, and $E_{1}$ and $E_{2}$ the corresponding edge sets defined in the proof of Lemma 3.

We now define for each $e \in E(G)$

$$
\theta_{e}=\left\{\begin{array}{lll}
0 & \text { if } & e \notin E_{1} \cup E_{2} \\
1 & \text { if } & e \in E_{1} \\
2 & \text { if } & e \in E_{2},
\end{array}\right.
$$


and for each $e \in E_{1} \cup E_{2}$

$$
\phi_{e}=\left\{\begin{array}{lll}
(0,1) & \text { if } & e \in E_{1} \\
(0,0) & \text { if } & e \in E_{2} .
\end{array}\right.
$$

The functions $\theta: E(G) \rightarrow\{0,1,2\}$ and $\phi: E\left(G_{\theta}\right) \rightarrow \mathbb{Z}_{2} \times \mathbb{Z}_{2}$ thus defined have the following properties : (a) since $G_{\theta}=H$, we have $\pi\left(A, G_{\theta}\right)=\pi(A, H)=\pi\left(A, G^{\prime}\right)=\pi$; (b) by its definition, $\phi$ satisfies Equation (15); (c) since the vertices of $A$ are the only vertices of $G_{\theta}=H$ incident with an odd number of edges of $E_{1}$ (cf. the proof of Lemma 3), $\phi$ satisfies Equation (21) with $B=\emptyset, C=A$. It follows from (b) and (c) that $\phi \in \Phi_{\theta}(\emptyset, A)$, i.e. $\Phi_{\theta}(\emptyset, A)$ is not empty, and from this fact together with (a) that $\theta \in \Theta_{\pi}(A)$, which proves the lemma.

It follows from Lemmas 1-3 that

$$
\left|\Phi_{\theta}(B, C)\right|=\left\{\begin{array}{l}
\left|\Phi_{\theta}(\emptyset, B \cup C)\right| \text { if } \pi\left(B, G_{\theta}\right) \quad \text { is an even partition } \\
0 \quad \text { otherwise. }
\end{array}\right.
$$

From Equations (12), (22), and (26) we conclude that

$$
\left(\sigma_{B}\right)_{G}\left(\sigma_{C}\right)_{G}=\sum_{\pi \in \Pi_{B}^{Q} \cup C(G)}\left\{\sum_{\theta \in \Theta_{\pi}(B \cup C)} 2^{2|V(G)|} \gamma_{\theta} g(\theta)\right\} \eta_{\pi}(B),
$$

where for any set $B \subset A$ and any partition $\pi \in \Pi_{A}$

$$
\eta_{\pi}(B)= \begin{cases}1 & \text { if the number of elements of } B \text { in every block of } \pi \text { is even } \\ 0 & \text { otherwise. }\end{cases}
$$

We observe that the factor in front of $\eta_{\pi}(B)$ in Equation (27) depends only on $B \cup C$, not on $B$ and $C$ separately. This implies that the function $A_{A}(G, K)$ introduced in Equation (6) can be written in the form

$$
\Lambda_{A}(G, K)=\sum_{\pi \in \Pi_{A}^{e}(G)}\left\{\sum_{\theta \in \Theta_{\pi}(A)} 2^{2|V(G)|} \gamma_{\theta} g(\theta)\right\}\left\{\sum_{B \subset A}^{e} \lambda_{B} \eta_{\pi}(B)\right\} .
$$

We now have the following two theorems:

Theorem 1. If $A$ is an even set of vertices of a finite graph $G$, and $\left\{\lambda_{B}\right\}_{B \subset A}^{e}$ a set of complex numbers defined for all even sets $B \subset A$, with $\lambda_{B}=\lambda_{A \backslash B}$ for all $B$, then

$$
\sum_{B \subset A}^{e} \lambda_{B}\left(\sigma_{B}\right)_{G}\left(\sigma_{A \backslash B}\right)_{G}=0
$$

for every Ising model on $G$ if and only if

$$
\sum_{B \subset A}^{e} \eta_{\pi}(B) \lambda_{B}=0
$$

for every partition $\pi \in \Pi_{A}^{e}(G)$.

Proof. If Equation (30) holds for all $\pi \in \Pi_{A}^{e}(G)$, then by Equation (28), $\Lambda_{A}(G, K)$ vanishes [i.e., Eq. (29) holds] for all $K \in \mathscr{K}$. Conversely, suppose that $\Lambda_{A}(G, K)$ vanishes for all $K \in \mathscr{K}$. Let $\pi$ be an arbitrary element of $\Pi_{A}^{e}(G), H$ a skeleton graph associated with $\pi, E_{1}$ and $E_{2}$ the edge sets and $\theta$ and $\phi$ the associated functions defined in the proofs of Lemmas 3 and 4. It was shown in these proofs that $\theta$ and $\phi$ 
satisfy Equations (15) and (21). On the other hand, if $\phi^{\prime}$ is a function on $E(H)$ satisfying Equation (21), the set of edges $e$ with $\phi_{e}^{\prime} \neq \phi_{e}$ must be a cycle of $H$. Since the only cycle of $H$ is the empty cycle, we have $\phi^{\prime}=\phi$, i.e., $\phi$ is the only function with domain $E(H)$ which satisfies Equation (21). Equation (15) then shows that the function $\theta$ defined by Equation (24) is the only element of $\Theta_{\pi}(A)$ such that $\theta_{e}=0$ for $e \in E(G) \backslash E(H)$, i.e. if $\theta^{\prime} \in \Theta_{\pi}(A)$ and $\theta^{\prime} \neq \theta$ then $\theta_{e} \neq 0$ for some $e \in E(G) \backslash E(H)$.

Let now $K_{0}$ be a positive real constant and $K$ the function on $E(G)$ defined by

$$
\begin{array}{lll}
K_{e}=K_{0} & \text { for } & e \in E(H) \\
K_{e}=0 & \text { for } & e \in E(G) \backslash E(H) .
\end{array}
$$

By Equation (10) we have in this case

$$
g(\theta)=\left(c_{0} s_{0}\right)^{\left|E_{1}\right|}\left(s_{0}^{2}\right)^{\left|E_{2}\right|},
$$

where $c_{0}=\cosh K_{0}, s_{0}=\sinh K_{0}$. On the other hand, we have $\gamma_{\theta}=1$. For any other function $\theta^{\prime} \in \Theta_{\pi}(A), g\left(\theta^{\prime}\right)$ contains at least one factor $c_{e} s_{e}$ or $s_{e}^{2}$ for $e \in E(G) \backslash E(H)$, and hence vanishes. The same applies to any other $\theta^{\prime} \in \Theta$ such that $E\left(G_{\theta^{\prime}}\right) \backslash E\left(G_{\theta}\right) \neq \emptyset$, in particular to any $\theta^{\prime} \in \Theta_{\pi^{\prime}}(A)$ where $\pi^{\prime}$ is not a refinement of $\pi$.

Consider now a partition $\pi^{\prime} \in \Pi_{A}^{e}(G)$ where $\pi^{\prime}(\neq \pi)$ is a refinement of $\pi$, and a function $\theta^{\prime} \in \Theta_{\pi^{\prime}}(A)$ such that $E\left(G_{\theta^{\prime}}\right) \subset E\left(G_{\theta}\right)$. From the definition of the set $E_{1}$ and the fact that $\pi^{\prime}$ is even it follows that $\theta^{\prime}$ is obtained from $\theta$ by replacing the value 2 by the value 0 for one or more properly chosen edges of $E_{2}$. Hence,

$$
g\left(\theta^{\prime}\right)=\left(c_{0} s_{0}\right)^{\left|E_{1}\right|}\left(s_{0}^{2}\right)^{\left|E_{2}\right|-r} \text { with } r \geqq 1 .
$$

It follows that $2^{-2|V(G)|}\left(c_{0} s_{0}\right)^{-\left|E_{1}\right|} \Lambda_{A}(G, K)$ is a polynomial in $s_{0}^{2}$ of degree $\left|E_{2}\right|$, in which the coefficient of the term of highest degree is $\sum_{B \subset A}^{e} \eta_{\pi}(B) \lambda_{B}$.

Since $\Lambda_{A}(G, K)=0$ for all values of the constant $K_{0}$, we must have

$$
\sum_{B \subset A}^{e} \eta_{\pi}(B) \lambda_{B}=0
$$

which completes the proof of the theorem.

Theorem 2. If $A$ is an even set of vertices of a finite graph $G$, and $\left\{\lambda_{B}\right\}_{B \subset A}^{e}$ a set of real numbers defined for all even sets $B \subset A$, with $\lambda_{B}=\lambda_{A \backslash B}$ for all $B$, then

$$
\sum_{B \subset A}^{e} \lambda_{B}\left(\sigma_{B}\right)_{G}\left(\sigma_{A \backslash B}\right)_{G} \geqq 0
$$

for every ferromagnetic Ising model on $G$ if and only if

$$
\sum_{B \subset A}^{e} \eta_{\pi}(B) \lambda_{B} \geqq 0
$$

for every partition $\pi \in \Pi_{A}^{e}(G)$.

Proof. For $K \in \mathscr{F}$ we have $g(\theta) \geqq 0$ for all $\theta \in \Theta$. Therefore, if Equation (35) holds for all $\pi \in \Pi_{A}^{e}(G)$, then, by Equation (28), $\Lambda_{A}(G, K)$ is nonnegative [i.e., Equation (34) holds] for all $K \in \mathscr{F}$. Conversely, suppose $\Lambda_{A}(G, K) \geqq 0$ for all $K \in \mathscr{F}$. Let $\pi$ be an arbitrary element of $\Pi_{A}^{e}(G)$. Following the lines of the proof of Theorem 1 we 
construct an interaction function $K \in \mathscr{F}$ for which $2^{-2|V(G)|}\left(c_{0} S_{0}\right)^{-\left|E_{1}\right|} \Lambda_{A}(G, K)$ is a polynomial in $s_{0}^{2}$. Since this polynomial is assumed to be nonnegative for all nonnegative values of $K_{0}$, the coefficient of the term of highest degree must be positive, i.e.

$$
\sum_{B \subset A}^{e} \eta_{\pi}(B) \lambda_{B} \geqq 0,
$$

which completes the proof of the theorem.

Corollary. If $A$ is an even set of vertices of a finite graph $G$, and $\left\{\lambda_{B}\right\}_{B \subset A}^{e}$ a set as defined in Theorem 2, then

$$
\sum_{B \subset A}^{e} \lambda_{B}\left(\sigma_{B}\right)_{G}\left(\sigma_{A \backslash B}\right)_{G}>0
$$

for every strictly ferromagnetic Ising model on $G$ if and only if Equation (35) holds for all $\pi \in \Pi_{A}^{e}(G)$ and in addition

$$
\sum_{B \subset A}^{e} \eta_{\pi}(B) \lambda_{B}>0
$$

for at least one $\pi \in \Pi_{A}^{e}(G)$.

Proof. The corollary follows immediately from Theorems 1 and 2 together with the fact that $\Lambda_{A}(G, K)$ is an (entire) analytic function of all coupling parameters.

\section{Generalization of Theorems 1 and 2}

Theorems 1 and 2 can be extended to identities and inequalities for correlation functions on a vertex set $A$ in which the products $\left(\sigma_{B}\right)\left(\sigma_{C}\right)$ refer to subsets $B$ and $C$ of $A$ which satisfy the condition that their symmetric difference (to be denoted by $B C$ ) is a given set $D \subset A$; the case discussed thus far, where $B$ and $C$ are disjoint and their union is $A$, corresponds to the choice $D=A$.

It is possible to derive these generalizations by a proper extension of the analysis of the preceding section. However, for reasons of transparency, and in order to show that the general case is, in a certain sense, already included in the special case $D=A$, we shall present another derivation, starting from the results of Section 2.

Let $A$ be a (not necessarily even) subset of a graph $G$, and $D$ an even subset of $A$. By $\Pi_{A}(D)$ we denote the set of all partitions of $A$ in which the number of vertices of $D$ in each block is even, and by $\Pi_{A}(D, G)$ the set of those partitions in $\Pi_{A}(D)$ that are induced by spanning subgraphs of $G$.

Theorem 1*. If $A$ is an arbitrary set of vertices of a finite graph $G, D$ an even subset of $A$, and $\left\{\lambda_{B}\right\}_{B \subset A}^{e}$ a set of complex numbers defined for all even sets $B \subset A$, with $\lambda_{B}=\lambda_{B D}$ for all $B$, then

$$
\sum_{B \subset A}^{e} \lambda_{B}\left(\sigma_{B}\right)_{G}\left(\sigma_{B D}\right)_{G}=0
$$

for every Ising model on $G$ if and only if

$$
\sum_{B \subset A}^{e} \eta_{\pi}(B) \lambda_{B}=0
$$

for all partitions $\pi \in \Pi_{A}(D, G)$. 
Proof. Let $Q=A \backslash D$, and $Q^{\prime}$ a set of vertices not in $V(G)$ which are in a one-to-one correspondence with the vertices of $Q$; the vertex in $Q^{\prime}$ corresponding to $v \in Q$ will be denoted by $v^{\prime}$. Let $G^{*}$ be the graph defined by $V\left(G^{*}\right)=V(G) \cup Q^{\prime}, E\left(G^{*}\right)$ $=E(G) \cup\left\{\left\{v, v^{\prime}\right\} \mid v \in Q\right\}$, and let $A^{*}=A \cup Q^{\prime}$. We extend the interaction function $K$ to a function $K^{*}$ on $E\left(G^{*}\right)$ by defining

$$
\begin{array}{ll}
K_{e}^{*}=K_{e} \quad \text { if } & e \in E(G) \\
K_{e}^{*}=K_{0}>0 & \text { if } \quad e \in E\left(G^{*}\right) \backslash E(G) .
\end{array}
$$

It is readily verified that for any set of complex numbers $\left\{\lambda_{B}\right\}_{B \subset A}^{e}$ we have,

$$
\sum_{B \subset A}^{e} \lambda_{B}\left(\sigma_{B}\right)_{G^{*}, K^{*}}\left(\sigma_{A^{*} \backslash B}\right)_{G^{*}, K^{*}}=\left(4 \cosh K_{0} \sinh K_{0}\right)^{|Q|} \sum_{B \subset A}^{e} \lambda_{B}\left(\sigma_{B}\right)_{G, K}\left(\sigma_{B D}\right)_{G, K} .
$$

It follows that the sum in the right-hand side of Equation (40) vanishes if and only if the left-hand side is zero, i.e., by Theorem 1 , if and only if $\sum_{B \subset A}^{e} \eta_{\pi^{*}}(B) \lambda_{B}=0$ for every $\pi^{*} \in \Pi_{A^{*}}^{e}\left(G^{*}\right)$; strictly speaking this requires the symmetrization of the set $\left\{\lambda_{B}\right\}_{B \subset A}^{e}$ with respect to $A^{*}$.

Consider a partition $\pi^{*} \in \Pi_{A^{*}}^{e}\left(G^{*}\right)$. Since $\pi^{*}$ is an even partition no vertex $v^{\prime} \in Q^{\prime}$ can form a block by itself. Therefore every vertex $v^{\prime} \in Q^{\prime}$ is in the same block as the corresponding vertex $v \in Q$; hence, the number of elements of $D$ in every block is even. Let now $\pi$ be the partition of $A$ obtained from $\pi^{*}$ by deleting all vertices of $Q^{\prime}$. Obviously, $\pi \in \Pi_{A}(D, G)$. Conversely, every $\pi \in \Pi_{A}(D, G)$ can be supplemented to a partition $\pi^{*} \in \Pi_{A^{*}}^{e}\left(G^{*}\right)$ by putting each vertex $v^{\prime} \in Q^{\prime}$ into the same block as the corresponding vertex $v \in Q$, and hence the theorem follows.

The corresponding generalization of Theorem 2 (to be referred to as Theorem $\left.2^{*}\right)$ is obvious and will not be discussed explicitly. Equation (38) and the analogous generalization of Equation (34) will again be called a $\Lambda$-identity and a $\Lambda$ inequality, respectively.

Theorems $1 *$ and $2 *$ remain valid if the unnormalized correlation functions are replaced by the corresponding normalized correlation functions, provided we restrict ourselves to Ising models with $Z \neq 0$; in Theorem $2^{*}$ this condition is always satisfied. The resulting identities and inequalities remain valid if for some edge $e=\{u, v\}$ we take the limit $K_{e} \rightarrow \infty$, i.e. if the edge $e$ is contracted and the spin variables $\sigma_{u}$ and $\sigma_{v}$ are identified.

Using Equations (28) and (40) and the relations

$$
\frac{\partial}{\partial K_{e}}\left(\sigma_{A}\right)=\left(\sigma_{A} \sigma^{e}\right), \frac{\partial g_{e}(1)}{\partial K_{e}}=1+2 g_{e}(2), \frac{\partial g_{e}(2)}{\partial K_{e}}=2 g_{e}(1),
$$

we also find that taking the derivative with respect to any coupling parameter $K_{e}(e \in E(G))$ in a $\Lambda$-identity ( $\Lambda$-inequality) results in a $\Lambda$-identity ( $\Lambda$-inequality). For $\Lambda$-inequalities this implies, in the terminology of Newman [5], that they apply strongly.

\section{Some Properties of Sets of $\boldsymbol{\Lambda}$-Identities}

It follows from Theorem 1 that for a given graph $G$ and a given set $A \subset V(G)$ the number of linearly independent $\Lambda$-identities, with $D=A$, for spin correlation 
functions on $A$, to be denoted by $L_{A}(G)$, is equal to the number of linearly independent solutions of the set of linear equations (30). The latter number depends on $G$ and $A$ only through the set of partitions $\Pi_{A}^{e}(G)$. In general, the larger the set $\Pi_{A}^{e}(G)$, and hence the number of conditions on $\lambda$, the smaller the number of linearly independent solutions of Equation (30), and hence $L_{A}(G)$. In particular, we have the following theorem.

Theorem 3. If $G$ and $G^{\prime}$ are finite graphs, and $A$ is an even subset of $V(G)$ and $V\left(G^{\prime}\right)$, then
i) $L_{A}(G)=0$
if $\quad \Pi_{A}^{e}(G)=\Pi_{A}^{e}$,
ii) $L_{A}\left(G^{\prime}\right) \leqq L_{A}(G)$ if $\Pi_{A}^{e}(G) \subset \Pi_{A}^{e}\left(G^{\prime}\right)$,
iii) $L_{A}(G)=2^{|A|-2} \quad$ if $\Pi_{A}^{e}(G)=\emptyset$.

Proof. i) If $\Pi_{A}^{e}(G)=\Pi_{A}^{e}$, Equation (30) is required to hold for all even partitions $\pi$ of $A$. We shall show that already the set of equations obtained by restricting $\pi$ to the partitions of $A$ into one or two even subsets has no nontrivial solution.

If $P_{\pi}$ and $A \backslash P_{\pi}$ are the subsets into which $A$ is partitioned by $\pi$ (where for convenience a one-block partition is considered as a partition of $A$ into $\emptyset$ and $A$ ), then for any even set $B C A$

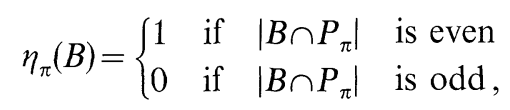

i.e.,

$$
\eta_{\pi}(B)=\frac{1}{2}\left(1+(-1)^{\left|B \cap P_{\pi}\right|}\right) \text {. }
$$

The set of equations (30), with the restriction imposed on $\pi$, can therefore be written as

$$
\sum_{B \subset A}^{e}\left(1+(-1)^{|B \cap P|}\right) \lambda_{B}=0
$$

for all even $P \subset A$; observe that the equations with $P$ and $A \backslash P$ are identical. Multiplying Equation (42) with $(-1)^{\left|B^{\prime} \cap P\right|}$, with $B^{\prime} C A,\left|B^{\prime}\right|$ even, summing over $P$ and using the relation

$$
\sum_{P \subset A}^{e}(-1)^{|U \cap P|}=2^{|A|-1}\left(\delta_{U, \emptyset}+\delta_{U, A}\right)
$$

for $U=B^{\prime}$ and $U=B B^{\prime}$, we obtain

$$
2^{|A|-1}\left[\left(\sum_{B \subset A}^{e} \lambda_{B}\right)\left(\delta_{B^{\prime}, \mathbb{b}}+\delta_{B^{\prime}, A}\right)+\left(\lambda_{B^{\prime}}+\lambda_{A \backslash B^{\prime}}\right)\right]=0 .
$$

It follows that $\lambda_{B^{\prime}}+\lambda_{A \backslash B^{\prime}}=0$ for all even sets $B^{\prime} \subset A$. Since we have taken $\lambda_{B^{\prime}}=\lambda_{A \backslash B^{\prime}}$, it follows that $\lambda_{B^{\prime}}=0$ for all even sets $B^{\prime} \subset A$. Hence, $L_{A}(G)=0$.

ii) If $\Pi_{A}^{e}(G) \subset \Pi_{A}^{e}\left(G^{\prime}\right)$, the set of linear equations (30) for the graph $G$ is a (proper or improper) subset of that of $G^{\prime}$, and hence the set of identities of the type (29) valid in $G^{\prime}$ is a subset of the set of identities valid in $G$. Statement ii) follows. 
iii) If $\Pi_{A}^{e}(G)=\emptyset$ there are no linear equations for the coefficients (except the condition $\lambda_{B}=\lambda_{A \backslash B}$ for all $B$ ), and we have $L_{A}(G)=\frac{1}{2} \mid\{B|B C A| B \mid$, even $\} \mid=2^{|A|-2}$. The simplest set of independent identities then consists of the equations $\left(\sigma_{B}\right)_{G}\left(\sigma_{A \backslash B}\right)_{G}=0$ for all $K \in \mathscr{K}(B \subset A,|B|$ even $)$, the validity of which is trivial.

Examples of the three cases considered in Theorem 3 are:

i) $G$ is a complete graph, i.e. $G=\left(V, E_{c}\right)$, with $E_{c}=\left\{\left\{v, v^{\prime}\right\} \mid v, v^{\prime} \in V, v \neq v^{\prime}\right\}$,

ii) $G$ is a spanning subgraph of $G^{\prime}$,

iii) $G$ is an empty graph, i.e. $G=(V, \emptyset)$.

If $G=(V, E)$ with $E \neq E_{c}$ there is at least one set $A \subset V$ for which $L_{A}(G)>0$, viz. $A=V$. This is expressed in the following lemma.

Lemma 5. If $G=(V, E)$ with $E=E_{c} \backslash e\left(e \in E_{c}\right)$ we have the following identity

$$
\sum_{B C V}^{e}(-1)^{\left|B \cap\left\{v_{1}, v_{2}\right\}\right|}\left(\sigma_{B}\right)_{G}\left(\sigma_{V \backslash B}\right)_{G}=0,
$$

where $v_{1}$ and $v_{2}$ are the vertices incident with $e$.

Proof. The equations (30) read in this case

$$
\sum_{B \subset V}^{e} \eta_{\pi}(B)(-1)^{\left|B \cap\left\{v_{1}, v_{2}\right\}\right|}=0
$$

or

$$
\sum_{R \subset V^{\prime \prime}}^{e} \eta_{\pi}\left(\left\{v_{1}, v_{2}\right\} \cup R\right)-\sum_{R \subset V^{\prime \prime}}^{o} \eta_{\pi}\left(\left\{v_{1}\right\} \cup R\right)=0,
$$

where $V^{\prime \prime}=V \backslash\left\{v_{1}, v_{2}\right\}$, and $\sum_{R \subset V^{\prime \prime}}^{o}$ denotes summation over all odd subsets of $V^{\prime \prime}$.

Consider first a partition $\pi$ in which $v_{1}$ and $v_{2}$ are in the same block. By the structure of $G$, partitions in which $v_{1}$ and $v_{2}$ form a block by themselves are not contained in $\Pi_{V}^{e}(G)$. Therefore, if we denote the blocks of $\pi$ by $U_{1}, U_{2}, \ldots, U_{r}$ (where $U_{1}$ is the block containing $v_{1}$ and $v_{2}$ ), $U_{1} \cap V^{\prime \prime}$ is not empty. The first sum on the left-hand side of (44) is equal to the number of even sets $R \subset V^{\prime \prime}$ such that $\left|R \cap U_{i}\right|$ is even for $1 \leqq i \leqq r$, the second sum is equal to the number of odd sets $R \subset V^{\prime \prime}$ such that $\left|R \cap U_{i}\right|$ is even for $2 \leqq i \leqq r$ and odd for $i=1$. Since $U_{1} \cap V^{\prime \prime}$ is not empty, the number of even subsets of $U_{1} \cap V^{\prime \prime}$ equals the number of odd subsets. Hence, the two sums in the left-hand side of (44) cancel. A similar argument applies to partitions in which $v_{1}$ and $v_{2}$ are in different blocks.

The generalization of Theorem 3 to the case that $A$ is an arbitrary vertex set of $G$ and $D$ an even subset of $A$ is straightforward.

\section{Examples of $\boldsymbol{\Lambda}$-Identities}

1) As a first example we discuss in detail the case $|A|=4, D=A$. Let $A$ $=\left\{v_{1}, v_{2}, v_{3}, v_{4}\right\}$. We have, with $\lambda_{i j}=\lambda_{\{i, j\}}$ and $\sigma_{i}=\sigma_{v_{i}}$,

$$
\begin{aligned}
\Lambda_{A}(G, K)= & 2\left[\lambda_{\vartheta}(1)\left(\sigma_{1} \sigma_{2} \sigma_{3} \sigma_{4}\right)+\lambda_{12}\left(\sigma_{1} \sigma_{2}\right)\left(\sigma_{3} \sigma_{4}\right)\right. \\
& \left.+\lambda_{13}\left(\sigma_{1} \sigma_{3}\right)\left(\sigma_{2} \sigma_{4}\right)+\lambda_{14}\left(\sigma_{1} \sigma_{4}\right)\left(\sigma_{2} \sigma_{3}\right)\right] .
\end{aligned}
$$

The set $\Pi_{A}^{e}(G)$ is a subset of the set $\Pi_{A}^{e}=\left\{\pi_{i} \mid 0 \leqq i \leqq 3\right\}$ where, in an obvious notation,

$$
\pi_{0}=(1234), \pi_{1}=(12 \mid 34), \pi_{2}=(13 \mid 24), \pi_{3}=(14 \mid 23) .
$$


If we define:

$$
k_{A}(\pi)=\frac{1}{2} \sum_{B \subset A}^{e} \lambda_{B} \eta_{\pi}(B),
$$

we find,

$$
\begin{aligned}
& k_{A}\left(\pi_{0}\right)=\lambda_{\emptyset}+\lambda_{12}+\lambda_{13}+\lambda_{14}, \\
& k_{A}\left(\pi_{1}\right)=\lambda_{\emptyset}+\lambda_{12}, \\
& k_{A}\left(\pi_{2}\right)=\lambda_{\emptyset}+\lambda_{13}, \\
& k_{A}\left(\pi_{3}\right)=\lambda_{\emptyset}+\lambda_{14} .
\end{aligned}
$$

a) If $\Pi_{A}^{e}(G)=\Pi_{A}^{e}$, then according to Theorem 3 there are no $A$-identities for $A$, as can easily be checked by putting all $k_{A}\left(\pi_{i}\right), 0 \leqq i \leqq 3$, equal to zero. As remarked above, this case is realized e.g. if $G$ is a complete graph, with $A \subset V(G)$.

b) We now consider the cases where $\left|\Pi_{A}^{e}(G)\right|=3$. One easily verifies that the only possibilities are $\Pi_{A}^{e}(G)=\Pi_{A}^{e} \backslash\left\{\pi_{i}\right\}$ with $1 \leqq i \leqq 3$, since $\pi_{0}$ is in $\Pi_{A}^{e}(G)$ whenever two of the three partitions $\pi_{i}(1 \leqq i \leqq 3)$ are in $\Pi_{A}^{e}(G)$. Without lack of generality we assume $\Pi_{A}^{e}(G)=\left\{\pi_{0}, \pi_{1}, \pi_{3}\right\}$. The condition for the existence of an identity now consists of the equations $k_{A}\left(\pi_{i}\right)=0(i=0,1,3)$, which have as the only solution $\lambda_{\theta}$ $=-\lambda_{12}=\lambda_{13}=-\lambda_{14}$. The resulting identity reads

$$
\text { (1) }\left(\sigma_{1} \sigma_{2} \sigma_{3} \sigma_{4}\right)-\left(\sigma_{1} \sigma_{2}\right)\left(\sigma_{3} \sigma_{4}\right)+\left(\sigma_{1} \sigma_{3}\right)\left(\sigma_{2} \sigma_{4}\right)-\left(\sigma_{1} \sigma_{4}\right)\left(\sigma_{2} \sigma_{3}\right)=0 \text {. }
$$

The graphs $G$ for which this identity holds are characterized by the fact that the partition $\pi_{2}$ of the set $A$ is not induced by any spanning subgraph of $G$. This implies that every chain between $v_{1}$ and $v_{3}$ separates $v_{2}$ from $v_{4}$ (i.e. has a vertex in common with every chain between $v_{2}$ and $v_{4}$ ).

The identity (48) is a special case of a general class of identities which formed the subject of $I$, and to which we shall return later on in this section (Example 4).

c) Consider now the cases where $\left|\Pi_{A}^{e}(G)\right|=2$. The only possibility is that where $\Pi_{A}^{e}(G)=\left\{\pi_{0}, \pi_{i}\right\}$ for some $i(1 \leqq i \leqq 3)$. Suppose $i=1$. From the equations $k_{A}\left(\pi_{0}\right)$ $=k_{A}\left(\pi_{1}\right)=0$ we find $\lambda_{\vartheta}=-\lambda_{12}, \lambda_{13}=-\lambda_{14}$, and hence

$$
\lambda_{\emptyset}\left[(1)\left(\sigma_{1} \sigma_{2} \sigma_{3} \sigma_{4}\right)-\left(\sigma_{1} \sigma_{2}\right)\left(\sigma_{3} \sigma_{4}\right)\right]+\lambda_{13}\left[\left(\sigma_{1} \sigma_{3}\right)\left(\sigma_{2} \sigma_{4}\right)-\left(\sigma_{1} \sigma_{4}\right)\left(\sigma_{2} \sigma_{3}\right)\right]=0
$$

for any $\lambda_{6}$ and $\lambda_{13}$. This implies

$$
\begin{aligned}
& (1)\left(\sigma_{1} \sigma_{2} \sigma_{3} \sigma_{4}\right)=\left(\sigma_{1} \sigma_{2}\right)\left(\sigma_{3} \sigma_{4}\right), \\
& \left(\sigma_{1} \sigma_{3}\right)\left(\sigma_{2} \sigma_{4}\right)=\left(\sigma_{1} \sigma_{4}\right)\left(\sigma_{2} \sigma_{3}\right) .
\end{aligned}
$$

This case applies when $G$ contains a vertex $v$ (cut vertex or articulation vertex) which separates $v_{1}$ and $v_{2}$ from $v_{3}$ and $v_{4}$ (i.e., which is contained in every chain between $v_{1}$ and $v_{3}$ etc.); $v$ need not be distinct from $v_{1}, v_{2}, v_{3}$ or $v_{4}$. This can be shown by introducing two new vertices $u$ and $u^{\prime}$, and four new edges $\left\{u, v_{1}\right\},\left\{u, v_{2}\right\}$, $\left\{u^{\prime}, v_{3}\right\},\left\{u^{\prime}, v_{4}\right\}$ and applying Menger's theorem to the vertices $u$ and $u^{\prime}$ (cf. [2, p. 129]).

The relations (50) are trivial in that they follow directly from the factorization of correlation functions in a graph with a cut vertex. 
d) If $\left|\Pi_{A}^{e}(G)\right|=1$ we have either $\Pi_{A}^{e}(G)=\left\{\pi_{0}\right\}$, or $\Pi_{A}^{e}(G)=\left\{\pi_{i}\right\}$ with $i=1,2$ or 3 ; suppose $i=1$. In both cases there are three linearly independent $\Lambda$-identities. In the former case we find

$$
\text { (1) }\left(\sigma_{1} \sigma_{2} \sigma_{3} \sigma_{4}\right)=\left(\sigma_{1} \sigma_{2}\right)\left(\sigma_{3} \sigma_{4}\right)=\left(\sigma_{1} \sigma_{3}\right)\left(\sigma_{2} \sigma_{4}\right)=\left(\sigma_{1} \sigma_{4}\right)\left(\sigma_{2} \sigma_{3}\right) \text {; }
$$

this case applies when $G$ contains a cut vertex $v$ (not necessarily distinct from $v_{1}, v_{2}, v_{3}$ or $\left.v_{4}\right)$ which separates every $v_{i}$ from every $v_{j}(1 \leqq i, j \leqq 4, i \neq j)$.

If $\pi_{1}$ is the only partition in $\Pi_{A}^{e}(G)$, we find

$$
\begin{aligned}
& (1)\left(\sigma_{1} \sigma_{2} \sigma_{3} \sigma_{4}\right)=\left(\sigma_{1} \sigma_{2}\right)\left(\sigma_{3} \sigma_{4}\right), \\
& \left(\sigma_{1} \sigma_{3}\right)\left(\sigma_{2} \sigma_{4}\right)=\left(\sigma_{1} \sigma_{4}\right)\left(\sigma_{2} \sigma_{3}\right)=0 .
\end{aligned}
$$

Here, $G$ is not connected, $v_{1}$ and $v_{2}$ are in one component and $v_{3}$ and $v_{4}$ in another one.

e) The case where $\Pi_{A}^{e}(G)$ is empty, has been dealt with in Theorem 3 .

2) We next consider the case $A=\left\{v_{1}, v_{2}, v_{3}, v_{4}\right\},|D|=2$, e.g. $D=\left\{v_{1}, v_{2}\right\}$. The set $\Pi_{A}(D, G)$ is a subset of the set $\{(1234),(123 \mid 4),(124 \mid 3),(12 \mid 34),(12|3| 4)\}$. Again, if $\Pi_{A}(D, G)$ contains all these partitions, there are no $\Lambda$-identities. In contrast with Example 1, the deletion of one partition from this maximum set does not necessarily lead to a $\Lambda$-identity. If, e.g., the partition (124|3) is missing (which is the case if $v_{3}$ separates $v_{1}$ and $v_{2}$ from $v_{4}$ ), one easily sees that the equations (39) have no non-trivial solution.

We discuss only one case in which identities do occur, viz. that where $v_{3}$ separates $v_{1}$ from $v_{2}$ and $v_{4}$. In that case, $\Pi_{A}(D, G)=\{(1234),(123 \mid 4)\}$. There are two conditions on the four independent coefficients $\lambda_{B}$, and we find the following identities,

$$
\begin{aligned}
& (1)\left(\sigma_{1} \sigma_{2}\right)=\left(\sigma_{1} \sigma_{3}\right)\left(\sigma_{2} \sigma_{3}\right), \\
& \left(\sigma_{1} \sigma_{4}\right)\left(\sigma_{2} \sigma_{4}\right)=\left(\sigma_{3} \sigma_{4}\right)\left(\sigma_{1} \sigma_{2} \sigma_{3} \sigma_{4}\right),
\end{aligned}
$$

which also follow, of course, from the factorization property mentioned above.

3) We now turn to the case $|A|=6, D=A$. If $A=\left\{v_{i} \mid 1 \leqq i \leqq 6\right\}$, then

$$
\Lambda_{A}(G, K)=2\left\{\lambda_{\emptyset}(1)\left(\sigma_{1} \sigma_{2} \sigma_{3} \sigma_{4} \sigma_{5} \sigma_{6}\right)+\sum_{\substack{i, j=1 \\ i<j}}^{6} \lambda_{i j}\left(\sigma_{i} \sigma_{j}\right)\left(\sigma_{i} \sigma_{j} \sigma_{A}\right)\right\} .
$$

We give one example of a $\Lambda$-identity which can occur in this case, viz. that with

$$
\begin{gathered}
\lambda_{6}=\lambda_{13}=\lambda_{24}=\lambda_{25}=\lambda_{26}=\lambda_{45}=\lambda_{46}=\lambda_{56}=1, \\
\lambda_{12}=\lambda_{14}=\lambda_{15}=\lambda_{16}=\lambda_{23}=\lambda_{34}=\lambda_{35}=\lambda_{36}=-1 .
\end{gathered}
$$

The corresponding numbers $k_{A}(\pi)$ are non-zero only for the partitions $\pi_{a}$ $=(13 \mid 2456), \pi_{b}=(13|24| 56), \pi_{c}=(13|25| 46)$ and $\pi_{d}=(13|26| 45)$. Hence, the corresponding $\Lambda$-identity holds if these partitions are not in $\Pi_{A}^{e}(G)$. This can occur in various ways, of which we mention the following ones.

a) $G$ is the graph obtained from the complete graph $K_{6}$ by deleting the edge $\left\{v_{1}, v_{3}\right\}$, and $A=V(G)$. This case has been dealt with in Lemma 5. 
b) $G$ is a planar graph and the sequence $\left(v_{1}, v_{2}, v_{3}, v_{4}, v_{5}, v_{6}\right)$ is a boundary sequence of $G$. A boundary sequence of a planar graph $G$ is a sequence of (not necessarily distinct) vertices $\left(u_{1}, \ldots, u_{n}\right)$ of $G$ such that the graph $G^{\prime}$ defined by $V\left(G^{\prime}\right)=V(G) \cup\left\{w_{0}, w_{1}, \ldots, w_{n}\right\} \quad\left(w_{i} \notin V(G), 0 \leqq i \leqq n\right)$ and $E\left(G^{\prime}\right)=E(G) \cup\left\{\left\{w_{0}, w_{i}\right\}\right.$, $\left.\left\{u_{i}, w_{i}\right\},\left\{w_{i}, w_{i+1}\right\} \mid 1 \leqq i \leqq n\right\}$ with $w_{n+1}=w_{1}$ is planar (see I). It was proven in I that for any boundary sequence $\left(u_{1}, u_{2}, u_{3}, u_{4}\right)$ every chain between $u_{1}$ and $u_{3}$ separates $u_{2}$ from $u_{4}$; furthermore, every subsequence of a boundary sequence is a boundary sequence. Applying these properties to the sequences $\left(v_{1}, v_{2}, v_{3}, v_{i}\right)(i=4,5,6)$ we see that indeed $\Pi_{A}^{e}(G)$ does not contain the partitions $\pi_{a}, \pi_{b}, \pi_{c}$, and $\pi_{d}$.

This example can be generalized, e.g. in the following way. Let $\left(v_{1}, v_{2}, v_{3}, v_{4}, w_{1}^{\prime}, v_{5}, v_{6}, w_{2}^{\prime}\right)$ be a boundary sequence of a planar graph $G_{1}, G_{2}$ an arbitrary graph such that $V\left(G_{1}\right) \cap V\left(G_{2}\right)=\left\{v_{5}, v_{6}, w_{1}^{\prime}, w_{2}^{\prime}\right\}$, and $G=G_{1} \cup G_{2}$, the union of $G_{1}$ and $G_{2}$. A chain between $v_{1}$ and $v_{3}$ which does not contain the vertices $v_{2}, v_{4}, v_{5}, v_{6}$ is either a chain in $G_{1}$ or it contains edges of $G_{2}$, and hence $w_{1}^{\prime}$ and $w_{2}^{\prime}$. In the former case it separates $v_{2}$ from $v_{4}, v_{5}$ and $v_{6}$; in the latter case it contains a chain between $w_{1}^{\prime}$ and $v_{3}$, which separates $v_{4}$ from $v_{2}, v_{5}$ and $v_{6}$. In both cases $\Pi_{A}^{e}(G)$ does not contain the partitions $\pi_{a}, \pi_{b}, \pi_{c}$, and $\pi_{d}$.

4) As a final example we give an alternative proof of the main result of $I$ mentioned in the introduction. Let $G$ be a finite planar graph, $S=\left(v_{1}, \ldots, v_{n}\right)$ a boundary sequence of $G, A$ the set of vertices occurring in $S$, and $D$ the set of vertices occurring an odd number of times. By Theorem $1^{*}$, the $\Lambda$-identity

$$
\sum_{j=1}^{n}(-1)^{j}\left(\sigma_{v_{1}} \sigma_{v_{j}}\right)\left(\sigma_{v_{1}} \sigma_{v_{j}} \prod_{k=1}^{n} \sigma_{v_{k}}\right)=0
$$

holds if and only if, for all partitions $\pi \in \Pi_{A}(D, G)$,

$$
-\eta_{\pi}(\emptyset)+\sum_{j=2}^{n}(-1)^{j} \eta_{\pi}\left(\left\{v_{1}, v_{j}\right\}\right)=0 .
$$

Let $\pi=\left\{U_{1}, \ldots, U_{l}\right\} \in \Pi_{A}(D, G)$, with $v_{1} \in U_{1}$. Then, for all $k(1 \leqq k \leqq l),\left|U_{k} \cap D\right|$ is even, and so the set $J_{k}:=\left\{j \mid v_{j} \in U_{k}, 1 \leqq j \leqq n\right\}$ is even. Let $J_{1}=\left\{j_{0}, j_{1}, \ldots, j_{m}\right\}$ with $j_{0}=1<j_{1}<\ldots<j_{m}$. Since $\eta_{\pi}\left(\left\{v_{1}, v_{j}\right\}\right)=1$ iff $v_{j} \in J_{1}$, and $\eta_{\pi}(\emptyset)=1$, Equation (56) reduces to

$$
\sum_{r=0}^{m}(-1)^{j_{r}}=0 .
$$

Since for any $r$ every chain between $v_{j_{r}}$ and $v_{j_{r+1}}$ separates all $v_{i}$ with $j_{r}<i<j_{r+1}$ from all other vertices of $S$, the set $\left\{\left.i\right|_{r}<i<j_{r+1}\right\}$ is a union of sets $J_{k}$, and hence even. Therefore, $(-1)^{j_{r}}=(-1)^{r-1}$, which proves the validity of Equation (56), and hence of Equation (55).

\section{Examples of $\Lambda$-Inequalities}

1) First consider the first GKS inequality [4], restricted to ferromagnetic Ising models on a graph, i.e. with pair interactions, which we write in the form

$$
\left(\sigma_{\emptyset}\right)\left(\sigma_{A}\right) \geqq 0 \text {. }
$$


Since $\eta_{\pi}(\emptyset)=1$ for all $\pi \in \Pi_{A}^{e}$, Equation (58) follows from Theorem 2 .

2) The second GKS inequality [4] for ferromagnetic Ising models reads

$$
\left(\sigma_{\emptyset}\right)\left(\sigma_{B} \sigma_{C}\right)-\left(\sigma_{B}\right)\left(\sigma_{C}\right) \geqq 0
$$

for arbitrary sets $B, C \subset V(G)$. The validity of this inequality in the case of pair interactions follows from the fact that $\eta_{\pi}(\emptyset)=1$ and $\eta_{\pi}(B)=0$ or 1 , and hence $\eta_{\pi}(\emptyset)$ $-\eta_{\pi}(B) \geqq 0$, for all $\pi \in \Pi_{B \cup C}(B C, G)$.

3) We next turn to a set of inequalities recently derived by Newman for ferromagnetic Ising models with pair interactions [5]. Let $G$ be a graph, $A \subset V(G)$, $A$ even, and $X_{A}$ a collection of even subsets of $A$ such that every partition of $A$ into pairs is a refinement of some two-block partition $(B \mid A \backslash B)$ of $A$ with $B \in X_{A}$. Newman's inequality reads

$$
\left(\sigma_{\emptyset}\right)\left(\sigma_{A}\right) \leqq \sum_{B \in X_{A}}\left(\sigma_{B}\right)\left(\sigma_{A \backslash B}\right)
$$

By Theorem 2, it is sufficient to prove that

$$
\eta_{\pi}(\emptyset)=1 \leqq \sum_{B \in X_{A}} \eta_{\pi}(B) \text { for all } \pi \in \Pi_{A}^{e}(G)
$$

Consider any $\pi \in \Pi_{A}^{e}(G)$ and let $\pi^{\prime}$ be a partition of $A$ into pairs which is a refinement of $\pi$. Since, by definition, there is at least one $B \in X_{A}$ such that $\pi^{\prime}$ is a refinement of $(B \mid A \backslash B)$ every block of $\pi^{\prime}$ contains 2 or 0 elements of $B$. Hence $\eta_{\pi^{\prime}}(B)=1$, and therefore $\eta_{\pi}(B)=1$. Since $\eta_{\pi}\left(B^{\prime}\right) \geqq 0$ for all other $B^{\prime} \in X_{A}$, the inequality (60) holds.

Our general formalism, applied to this particular case, resembles the derivation of Equation (60) given by Sylvester [6].

4) Finally we derive a new $\Lambda$-inequality for the case $|A|=6, D=A$, namely:

$$
\text { (1) }\left(\sigma_{A}\right)+\sum_{j=2}^{6}\left(\sigma_{1} \sigma_{j}\right)\left(\sigma_{1} \sigma_{j} \sigma_{A}\right) \leqq \sum_{\substack{i, j=2 \\ i<j}}^{6}\left(\sigma_{i} \sigma_{j}\right)\left(\sigma_{i} \sigma_{j} \sigma_{A}\right)
$$

By Theorem 2 it is sufficient to show that for all $\pi \in \Pi_{A}^{e}$

$$
1+\sum_{j=2}^{6} \eta_{\pi}\left(\left\{v_{1}, v_{j}\right\}\right) \leqq \sum_{\substack{i, j=2 \\ i<j}}^{6} \eta_{\pi}\left(\left\{v_{i}, v_{j}\right\}\right)
$$

If $\pi=(123456)$, we have $\eta_{\pi}\left(\left\{v_{i}, v_{j}\right\}\right)=1$ for all $i, j(i \neq j)$ and hence Equation (63) is valid. If $\pi$ is of the type (1j|klmn), the left-hand side of Equation (63) is equal to 2, the right-hand side to 6, and again Equation (63) is valid. The remaining two cases, where $\pi$ is of the type $(1 j k l \mid m n)$ or $(1 j|k l| m n)$, are dealt with in a similar way. The extension of the inequality (62) to the case $|A|=n \geqq 6, n$ even, is straightforward.

The examples of $\Lambda$-inequalities discussed above are valid for any graph $G$ containing the given sets $A$ and $D$. Evidently for any choice of $A$ and $D$, Theorem $2 *$ enables one in principle to derive all $\Lambda$-inequalities on $A$ which have the same general validity. The corresponding sets $\lambda$, considered as vectors in $R^{n}\left(n=2^{|A|-2}\right)$ 
with components $\lambda_{B}$, form a convex cone. If, for a particular choice of $G, \Pi_{A}(D, G)$ is a proper subset of $\Pi_{A}(D)$, the corresponding set of $\Lambda$-inequalities will again form a convex cone, which may contain the convex cone mentioned above as a proper subset.

In a subsequent paper [7] we shall investigate the structure of these convex cones. In particular we shall show that every $\Lambda$-inequality can be "decomposed" into a finite number of extremal inequalities. We shall discuss the relation of the inequalities mentioned in the examples with these extremal inequalities, and derive new (extremal) inequalities.

\section{Concluding Remarks}

1) Formally, the analysis of this paper is restricted to Ising models in zero magnetic field. The case of an arbitrary (not necessarily homogeneous) magnetic field can, however, be easily included by replacing the field by a "dummy" spin interacting with all other vertices [8].

2) Theorems $1\left(1^{*}\right), 2\left(2^{*}\right)$, and 3 have been derived for finite graphs. The extension to infinite graphs is straightforward. Consider an infinite graph $G$, two finite sets of vertices $A \subset V(G)$ and $D \subset A$, and for each partition $\pi \in \Pi_{A}^{e}(G)$ a skeleton graph $H_{\pi}$. The edge sets of all $H_{\pi}$ are finite. Hence there exists a finite subgraph $G_{0}$ of $G$ such that $E\left(H_{\pi}\right) \subset E\left(G_{0}\right)$ for all $\pi$. Let $G_{0}, G_{1}, G_{2}, \ldots$ be a sequence of increasing subgraphs of $G$ such that $\lim _{n \rightarrow \infty} G_{n}=G$ and that $\left\langle\sigma_{B}\right\rangle_{G}=\lim _{n \rightarrow \infty}\left\langle\sigma_{B}\right\rangle_{G_{n}}$ exists for all $B \subset A$. Then $\Pi_{A}^{e}\left(G_{n}\right)=\Pi_{A}^{e}(G)$ for $n=0,1,2, \ldots$, and the validity of the theorems (with the unnormalized correlation functions replaced by normalized correlation functions) for infinite graphs follows.

3) As the reader may have observed, all examples of $\Lambda$-identities given in Section 5 have the property that all $\lambda_{B}$ are equal to $1,-1$ or 0 . In a forthcoming paper [9], devoted to $\Lambda$-identities for Ising models with general ( $n$-spin) interactions, it will be shown that for every choice of $G, A$, and $D$ the set of all $\Lambda$-identities can be derived from an independent set of $\Lambda$-identities having this property.

4) Many theorems on inequalities include a specification of the condition under which the equality sign holds (cf., e.g., [10]). For correlation-function inequalities such conditions have hitherto not received much attention in the literature (see, however, [11]). In the case of $\Lambda$-inequalities they are implicit in Theorem $1 *$, since the validity of a $\Lambda$-equality for all $K \in \mathscr{F}$ implies the validity for all $K \in \mathscr{K}$. The condition takes the form of a condition on $\Pi_{A}(D, G)$. For the second GKS inequality, e.g., the condition reads (cf. Section 6, Example 2): $\eta_{\pi}(B)=1$ for all $\pi \in \Pi_{B \cup C}(B C, G)$, i.e. all partitions of $B \cup C$ for which $\eta_{\pi}(B)=0$ are absent from $\Pi_{B \cup C}(B C, G)$. E.g., if $B=\left\{v_{1}, v_{2}\right\}, C=\left\{v_{3}, v_{4}\right\}$, the missing partitions are (13|24) and (14|23), in which case $G$ contains a cut vertex separating $v_{1}$ and $v_{2}$ from $v_{3}$ and $v_{4}$ (see Section 5, Example 1c). In case $G$ contains a "dummy" vertex $v_{0}$ connected with all other vertices, representing a nonzero magnetic field, $v_{0}$ must be the cut vertex. The graph obtained from $G$ by deleting $v_{0}$ and the edges incident with it then has $v_{1}$ and $v_{2}$ in one component, $v_{3}$ and $v_{4}$ in another. This specific result was earlier derived by Setô [11]. 


\section{References}

1. Groeneveld,J., Boel, R.J., Kasteleyn,P.W.: Physica (to be published)

2. Wilson, R.J.: Introduction to graph theory. Edinburgh: Oliver and Boyd 1972

3. Harary,F.: Graph theory, pp. 37-38. Reading, Mass.: Addison-Wesley 1969

4. Griffiths, R. B.: Rigorous results and theorems. In: Phase transitions and critical phenomena, Vol. 1 (eds. C. Domb, M. S. Green), pp. 72-74. London: Academic Press 1972

5. Newman,C.: Z. Wahrscheinlichkeitstheorie verw. Gebiete 33, 75 (1975)

6. Sylvester, G. S.: Commun. math. Phys. 42, 209 (1975)

7. Kasteleyn,P.W., Boel, R.J.: (to be published)

8. Kasteleyn,P.W.: Graph theory and crystal physics. In: Graph theory and theoretical physics (ed. F. Harary), p. 43. London: Academic Press 1967 Griffiths, R. B.: J. Math. Phys. 8, 484 (1967)

9. Boel,R.J., Kasteleyn, P. W.: Physica (to be published)

10. Beckenbach,E.F., Bellman, R.: Inequalities. Berlin-Heidelberg-New York: Springer 1961

11. Setô, N.: Progr. Theor. Phys. 55, 683 (1976)

Communicated by E. Lieb

Received March 24, 1978 